\title{
On Geoneutrinos
}

L. B. Bezrukov ${ }^{1, \star}$, A. S. Kurlovich ${ }^{1}$, B. K. Lubsandorzhiev ${ }^{1}$, V. V. Sinev ${ }^{1}$, V. P. Zavarzina ${ }^{1}$, and V. P. Morgalyuk ${ }^{2}$

${ }^{1}$ Institute for Nuclear Researches of Russian Academy of Sciences, Prospekt 60-letia Oktyabrya 7a, Moscow, 115409, Russia

${ }^{2}$ A. N. Nesmeyanov Institute of Organoelement Compounds of Russian Academy of Sciences, Vavilova 10, Moscow, 115409, Russia

\begin{abstract}
Experimental data on geoneutrinos allow to admit that masses of $\mathrm{U}$, Th and $\mathrm{K}$ in the Earth can be up to $m_{\mathrm{U}}=1.7 \cdot 10^{17} \mathrm{~kg}, m_{\mathrm{Th}}=6.7 \cdot 10^{17} \mathrm{~kg}$ and $m_{\mathrm{K}} / m_{\text {Earth }} \sim 2 \%$. These values correspond to intrinsic Earth heat flux in $~ 300$ TW. The most part of this flux goes up in rift zones as a heated gases. Argo Project results and the measurements of the Moon intrinsic heat flux support the existence of such a big flux. So large of $U$, Th, $\mathrm{K}$ abundances were predicted by Adjusted Hydridic Earth model.
\end{abstract}

\section{Introduction}

The connection of geoneutrinos and Earth thermal flux was known for a long time. Detecting of geoneutrinos on the surface can give the exact value of radiogenic part of all heat produced inside the Earth [1]. Thermal flux is obtained by measuring the temperature gradient in the crust. The most exact value $47 \pm 2 \mathrm{TW}$ is obtained by the averaging of experimentally measured points. This method includes only part of the energy passing through the crust by conductivity way and does not take into account other possible ways of energy transfer. For example, the formation of gases could take some heat and decrease the conductivity termal flux. Gas may pass the crust and release the energy somewhere in the ocean.

We considered a number of possible Earth thermal flux values and concluded that value of order $300 \mathrm{TW}$ is preferable. This value may be easily explained if to admit the abudances of potassium and uranium with thorium in larger amounts inside of the Earth core. We proposed here the Adjusted Hydridic Earth model which can explain as the value of thermal flux in 300 TW and also the measured number of events in ongoing geoneutrino experiments.

\section{Earth models}

The Bulk Silicat Earth (BSE) model [2] gives:

$$
\begin{aligned}
m_{B S E}(\mathrm{U}) & =0.81 \cdot 10{ }^{17} \mathrm{~kg}, \\
m_{B S E}(\mathrm{Th}) & =3.16 \cdot 10{ }^{17} \mathrm{~kg}, \\
m_{B S E}\left({ }^{40} \mathrm{~K}\right) & =0.58 \cdot 10{ }^{17} \mathrm{~kg} .
\end{aligned}
$$

^e-mail: bezrukov@inr.ac.ru 
Table 1. Geochemical model of modern Hydridic Earth

\begin{tabular}{|c|c|c|}
\hline Geosphere & Depth range, km & Composition \\
\hline Lithosphere & $0-150$ & $\begin{array}{l}\mathrm{CaO}, \mathrm{MgO}, \mathrm{Al}_{2} \mathrm{O}_{3}, \mathrm{SiO}_{2}, \mathrm{Na}_{2} \mathrm{O}, \\
\mathrm{FeO}, \mathrm{H}_{2} \mathrm{O} \ldots\end{array}$ \\
\hline Astenosphere & 150 & $\begin{array}{l}\text { Thin layer of Metalsphere } \\
\text { with high hydrogen concentration }\end{array}$ \\
\hline Metal & $150-2900$ & $\mathrm{Mg}_{2} \mathrm{Si}: \mathrm{Si}: \mathrm{FeSi}=6: 3: 1$ \\
\hline External core & $2900-5000$ & $\begin{array}{l}\mathrm{MgH}_{0.1}, \mathrm{SiH}_{0.1}, \mathrm{FeH}_{0.1} \\
+\mathrm{MgH}_{\mathrm{n}}, \mathrm{SiH}_{\mathrm{n}}, \mathrm{FeH}_{\mathrm{n}}(\mathrm{n}=7)\end{array}$ \\
\hline Inernal core & $5000-6371$ & $\mathrm{MgH}_{\mathrm{n}}, \mathrm{SiH}_{\mathrm{n}}, \mathrm{FeH}_{\mathrm{n}}(\mathrm{n}=7)$ \\
\hline
\end{tabular}

This amount distributed only in the Crust and Upper Mantle in framework of BSE model.

There is an alternative Earth model [3], [4] named Hydridic Earth (HE) model which predicts the primordial chemical elements composition of the Earth.

Vladimir Larin [3] used the idea that the separation of the chemical elements in the solar system (chemical differentiation) was originated from the magnetic field of the Protosun. He found the law:

$$
\left(\frac{X_{M}}{X_{\mathrm{Si}}}\right)_{\text {Planet }}=\left(\frac{X_{M}}{X_{\mathrm{Si}}}\right)_{\text {Sun }} \cdot F\left(R_{\text {Planet }}, E_{I P}(M)\right)
$$

where $X_{M}$ is the mass fraction in the planet mass of the chemical element with atomic number $M, E_{I P}(M)$ is the ionization potential of the chemical element with atomic number $M$ in $\mathrm{eV}, R_{\text {Planet }}$ is the Planet distance to the Sun. In the work [4] the function F was proposed for the Earth in the form:

$$
F\left(R_{\text {Earth }}, E_{I P}(M)\right)=12100 \cdot e^{-1.1537 \cdot E_{I P}(M)} .
$$

We obtained from (2) and (3):

$$
\begin{aligned}
m_{H E}(\mathrm{U}) & =3.18 \cdot 10^{17} \mathrm{~kg}, \\
m_{H E}(\mathrm{Th}) & =1.05 \cdot 10^{18} \mathrm{~kg}, \\
m_{H E}\left({ }^{40} \mathrm{~K}\right) & =2.63 \cdot 10^{19} \mathrm{~kg} .
\end{aligned}
$$

by using experimental data of $\left(\frac{X_{M}}{X_{\mathrm{Si}}}\right)_{\text {Sun }}$ from [5] for the Sun and the data for $E_{I P}$ from [6]. Function $F$ from (3) is the theoretical one and can be adjusted.

The $18.3 \%$ of the Earth primordial mass is predicted to be Hydrogen [4]. The inner Earth would have been and still could be hydrogen rich. The most part of primordial hydrogen have escaped to atmosphere and space through the degassing of the mantle. Model suggests that large amounts of hydrogen are still located in the core.

Hydridic Earth model predicts huge amount of ${ }^{40} \mathrm{~K}$ in the Earth.

\section{Experimentally measured uranium and thorium geoneutrinos}

Geoneutrinos were detected with two experimental setups for the moment. They are: Borexino detector [7] constructed for the ${ }^{7}$ Be solar neutrinos registartion placed in Gran Sasso Laboratory and KamLAND detector [8] for testing neutrino oscillation parameters in solar region placed in Kamioka Observatory. Both detectors discovered geoneutrino flux that are in accordance with predicted values. Prediction is based on BSE model. On figure 1 antineutrino spectra are shown for three main isotopes emitting geoneutrinos. It is seen that only part of spectrum can be detected for ${ }^{238} \mathrm{U}$ and ${ }^{232} \mathrm{Th}$ using 


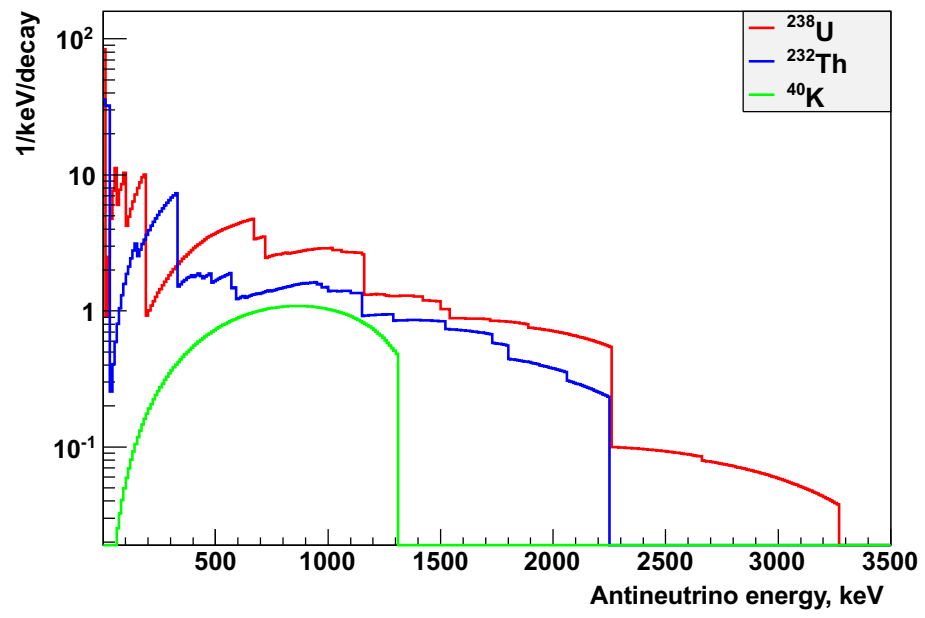

Figure 1. Antineutrino spectra from ${ }^{238} \mathrm{U},{ }^{232} \mathrm{Th}$ and ${ }^{40} \mathrm{~K}$ per decay. On $\mathrm{X}$ axis the antineutrino energy and on $\mathrm{Y}$ axis the number of neutrinos emitted per keV energy bin in one decay are shown.

Table 2. Counting rates of Borexino and KamLAND

\begin{tabular}{lcccc}
\hline Detector & $T_{\text {meas }}$, days & $N_{p}$ & $N_{\text {event }}$ & $R, \mathrm{TNU}^{*}$ \\
\hline Borexino & 2056 & $(0.977 \pm 0.05) \times 10^{31}$ & $23.7_{-5.7(s t)+0.8}^{+6.5(t)-0.6}$ & 43.5 \\
KamLAND & 2991 & $(5.98 \pm 0.13) \times 10^{31}$ & $116_{-27}^{+28}$ & 23.7 \\
\hline
\end{tabular}

${ }^{*} \mathrm{TNU}=1$ event per $10^{32}$ protons per year

the inverse beta-decay reaction on proton. This reaction has a threshold $1.8 \mathrm{MeV} .{ }^{40} \mathrm{~K}$ spectrum is totally under this threshold.

Counting rates of geoneutrinos are shown in table 2. From the table one can see that counting rate of geoneutrinos is extremely low for both detectors. It does not contradict to any Earth model, better to say it agrees with all possible models. To check models and distinguish between them one needs to accumulate sufficient statistics (4000-5000 events). Easily to estimate that Borexino needs to be operatable at least 1000 years to achieve this statistics. The only one way for investigation of geoneutrino fluxes is to develope a special detector having target mass no less than $10 \mathrm{kt}$ and low background.

\section{Earth thermal flux}

Thermal flux of the Earth is known for a long time. It is considered that the flux is produced by decays of long lived radioactive elements such as ${ }^{238} \mathrm{U},{ }^{232} \mathrm{Th}$ and ${ }^{40} \mathrm{~K}$. According to BSE model (1) the total radiogenic thermal flux is about $15 \mathrm{TW}$. Other part of the flux is considered to be produced by a number of not radioactive reasons [2]. Let consider the possible values of thermal flux that are known today. 


\subsection{Conductive part of heat flux}

The value of Earth thermal flux was measured by installing of thermosensors in the drilled holes all over the world in cotinental and ocean crust [9]. The data were averaged and some methods of data interpolation were used. As a result the value $47 \pm 2 \mathrm{TW}$ was obtained. It is nesessary to stress that this value was changing from 30 to $47 \mathrm{TW}$ during 2000-2010 years with changing of the interpolation methods.

This method does not account the possible ways of thermal transmission other than thermoconductivity.

\subsection{Heat transfer in oceans}

Energy imbalance in oceans was observed by use of monitoring temperature profile down to $2 \mathrm{~km}$ of depth in a numerous points. It is so called the ARGO project[10]. By monitoring of temperature during 6 years and using about 4000 special devices floating in different parts of world ocean they found that the accumulated energy in $2 \mathrm{~km}$ layer of ocean corresponds to $\sim 5 \times 10^{22} \mathrm{~J}$. This fact can be interpreted that ocean was heated this period mostly by intrinsic Earth source of heat. The value of thermal flux from this source must be $300 \mathrm{TW}$.

\subsection{Earth thermal flux recalculated from the measured one on the Moon}

Interpretation of surface heat flow is considerably less ambiguous on the Moon becauce is thought to be directly related to the abundance of radioactive elements. If the Moon and the Earth consisted of the same elements the thermal flux of the Earth is proportionally larger than the one of the Moon with coefficient of masses ratio. For the moment we know three measurements of Moon thermal flux: measurements by Apollo 15 and 17 missions [11], registering of radio waves from the Moon of $10 \mathrm{~cm}$ and $20 \mathrm{~cm}$ wavelengths [12] and measuring of surface temperature at the permanent dark places on Moon surface on the Moon south pole region [13], [14]. According Apollo measurements the Earth flux is appeared 43-65 TW. Second method gives the value about $170 \mathrm{TW}$. Third method gives the largest value $420 \mathrm{TW}$.

\section{Potassium abundance problem}

BSE model does not give the large value of potassium abundance in total Earth mass. It is considered that all potassium is concentrated in the crust. Potassium abundance in the crust is about $2 \%$ in weight. But the absence of potassium inside of Earth is based on the fact that meteorites have low abundance of potassium. In reality we have no information about potassium abundance inside the Earth below the crust. If its abundance in the Earth is about the same as in the crust the thermal flux could be much more larger than predicted BSE model. The only way to obtain information on the potassium inside the Earth is to measure antineutrino and possibly neutrino fluxes from ${ }^{40} \mathrm{~K}$ which content in natural potassium is $1.17 \times 10^{-4}$.

Calculated heat flux from ${ }^{40} \mathrm{~K}$ is ten times larger than one from ${ }^{238} \mathrm{U}$ and ${ }^{232} \mathrm{Th}$ in sum in case of its abundance on the level of $2 \%$ all over the Earth. From previous section where we discussed possible values of thermal flux we showed that Earth termal flux might be up to $300 \mathrm{TW}$.

Thermal flux from ${ }^{40} \mathrm{~K}$ can be nondirectly checked by registering antineutrino and neutrino fluxes produced by ${ }^{40} \mathrm{~K}$. In [15],[16] the spectrum of recoiled electrons from ${ }^{40} \mathrm{~K}$ was compared with spectra from other neutrino sources in Borexino detector. 


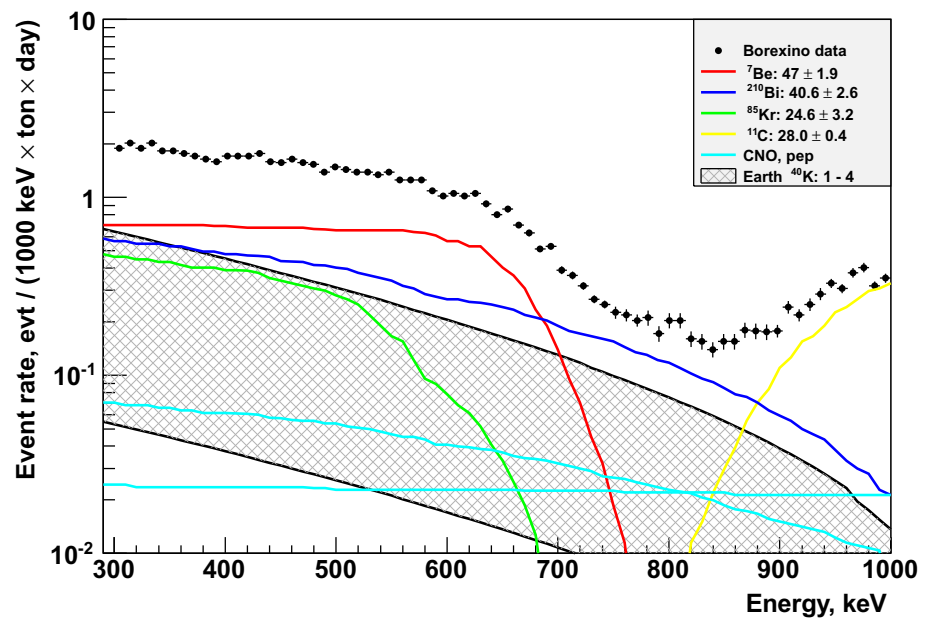

Figure 2. Area of ${ }^{40} \mathrm{~K}$ spectrum and solar neutrino spectra (pep and CNO). Main background spectra are also shown. On $\mathrm{X}$ axis the energy released per single event in the detector and on $\mathrm{Y}$ axis the event number per 1000 $\mathrm{keV}$ energy range per day in one ton of a target are shown. Numbers show the count rate per day from a source.

\section{Adjusted Hydridc Earth model.}

Can we explain the results of geoneutrino flux measurements and the value of heat flux derived from Argo experiment in the frame of HE model?

We propose the following adjusted HE (AHE) model. We took the function $F$ from (3) about twice less in the region of small $E_{I P}$. It gives us $m_{A H E}(\mathrm{U})=1.7 \cdot 10^{17} \mathrm{~kg}, m_{A H E}(\mathrm{Th})=6.7 \cdot 10^{17} \mathrm{~kg}$, $m_{A H E}\left({ }^{40} \mathrm{~K}\right)=1.2 \cdot 10^{19} \mathrm{~kg}$.

The equation relating masses and heat production is

$$
H=m \cdot \frac{N_{\text {Avog }}}{A} \cdot \frac{E_{\text {release }}}{\tau} \cdot \alpha,
$$

where $N_{A v o g}-$ Avogadro number, $A$ - atomic number, $E_{\text {release }}-$ energy release in decay chain in $\mathrm{MeV}, \tau=t_{1 / 2} / \ln 2-$ mean lifetime of isotope, $\alpha$ - the conversion factor $1 \mathrm{MeV}=1.602 \cdot 10^{-13} \mathrm{~J}$.

$$
\begin{array}{r}
H(\mathrm{U})=1.7 \cdot 10^{20}[\mathrm{~g}] \frac{6 \cdot 10^{23}}{238}\left[\mathrm{~g}^{-1}\right] \cdot \frac{47.7[\mathrm{MeV}]}{6.45 \cdot 10^{9} \cdot 3.15 \cdot 10^{7}[\mathrm{~s}]} \cdot 1.602 \cdot 10^{-13}[\mathrm{~J}] \\
=16.1 \mathrm{TW}, \\
\begin{aligned}
H(\mathrm{Th})=10.5 \cdot 10^{20}[\mathrm{~g}] \frac{6 \cdot 10^{23}}{232}\left[\mathrm{~g}^{-1}\right] \cdot \frac{40.4[\mathrm{MeV}]}{2.03 \cdot 10^{10} \cdot 3.15 \cdot 10^{7}[\mathrm{~s}]} \cdot 1.602 \cdot 10^{-13}[\mathrm{~J}] \\
=18.0 \mathrm{TW},
\end{aligned}
\end{array}
$$


Table 3. Number of events in modern detectors for $1 \mathrm{kt} \cdot$ year exposition

$$
\begin{aligned}
& \begin{array}{ccc}
\hline \text { Site } & \begin{array}{c}
\text { Experimental } \\
\text { count rate }
\end{array} & \begin{array}{c}
\text { Calculated } \\
\text { one }
\end{array} \\
\hline \text { Borexino } & 43.5 \pm 12.5 & 38.1 \\
\hline \text { KamLand } & 23.67 \pm 5.61 & 31.8 \\
\hline
\end{array} \\
& H\left({ }^{40} \mathrm{~K}\right)=1.2 \cdot 10^{22}[\mathrm{~g}] \frac{6 \cdot 10^{23}}{40}\left[\mathrm{~g}^{-1}\right] \cdot \frac{0,89 \cdot 0.598[\mathrm{MeV}]}{1.8 \cdot 10^{9} \cdot 3.15 \cdot 10^{7}[\mathrm{~s}]} \cdot 1.602 \cdot 10^{-13}[\mathrm{~J}]= \\
& 270 \text { TW. } \\
& H=H(\mathrm{U})+\mathrm{H}(\mathrm{Th})+\mathrm{H}\left({ }^{40} \mathrm{~K}\right)=304 \mathrm{TW} .
\end{aligned}
$$

This heat value is produced mostly by ${ }^{40} \mathrm{~K}$ decay and is consistent with Argo experiment results [10].

The experimental signal of the geoneutrino detectors depends not only on $m(\mathrm{U})$ and $m(\mathrm{Th})$ but also on the distribution of $U$ and Th in the Earth's interior. The calculations show that decays in the crust matter surrounding of the detector give the main contribution to the experimental signal [2]. The Hydridic Earth model predicts the existence of the Earth hydrogen degassation process. Hydrogen appears on the surface of the Earth core and goes up to the cosmic space through the long chain of processes. During this way the hydrogen flow purifies the metallic mantel volume up to lithosphere. So $\mathrm{U}$ and Th must locate in the present-day Earth mostly in the core and the lithosphere. The Internal core of present-day Earth still could be primordial and could have the primordial U and Th densities. The existence in the present day Earth core of the large amount of $U$ and Th is natural in the frame of the Hydridic Earth model.

We took for the crust $U$ and Th distribution according to BSE model and for mantel and core the concentrations of $U$ and $T h$ were chosen to satisfy the observed counting rates (Borexino and KamLAND). The results of calculation of geoneutrino detector counting rate for Borexino and KamLAND in the frame of AHE model are given in Table 3.

We demonstrated that Borexino and KamLAND results and Argo experiment result can be understood in the frame AHE model.

\section{Conclusion}

1. We found that the total Earth thermal flux might be larger than one measured using the temperature gradient.

2. We proposed Adjusted Hydridic Earth model with $m_{A H E}(\mathrm{U})=1.7 \cdot 10^{17} \mathrm{~kg}, m_{A H E}(\mathrm{Th})=$ $6.7 \cdot 10^{17} \mathrm{~kg}, m_{A H E}\left({ }^{40} \mathrm{~K}\right)=1.2 \cdot 10^{19} \mathrm{~kg}$. This model can explain the observed geoneutrino fluxes and the high thermal flux.

3. The detector with a target $10 \mathrm{kt}$ is needed to accumulate at least 4000-5000 geoneutrino events in 10-20 years of operation time. Directional analysis could clarify the question: Has the core some radioactivity or not? Extra pure scintillator allows to measure ${ }^{40} \mathrm{~K}$ spectrum and estimate the potassium amount in the whole Earth. This will make understandable the total Earth thermal flux. For better separation of ${ }^{40} \mathrm{~K}$ spectrum and neutrinos from $\mathrm{CNO}$ cycle it is very desirable to have independent measurement of CNO neutrinos. 


\section{Acknowledgements}

We are grateful to Vladimir Larin and Stefan Schoenert for useful discussions. This work was supported by grant of Russian Foundation of Basic Research No 13-02-92440.

\section{References}

[1] Livia Ludhova (Ed.), Geo-neutrinos (Open Academic Press, Berlin, Germany, 2016)

[2] Gianni Fiorentini, Marcello Lissia and Fabio Mantovani, Phys. Rept. 453, 117 (2007); arXiv:0707.3203 [physics.geo-ph]; Fabio Mantovani, Luigi Garmignani, Gianni Fiorentini and Marcello Lissia, arXiv:hep-ph/030913v2

[3] V. N. Larin (ed. C. Warren Hunt), Hydridic Earth: the New Geology of Our Primordially Hydrogen-Rich Planet (Polar Publishing, Calgary, Alberta, Canada, 1993) 247.

[4] Herve Toulhoat, Valerie Beaumont, Viacheslav Zgonnik, Nikolay Larin and Vladimir N. Larin, arXiv:1208.2909 [astro-ph.EP]

[5] Martin Asplund, Nicolas Grevesse and Jacques Sauval, Nucl.Phys. A 777, 1 (2006); arXiv:astro$\mathrm{ph} / 0410214 \mathrm{v} 2$

[6] David R. Lich (ed), CRC Handbook of Chemistry and Physics (84th Edition. CRC Press. Boca Raton, Florida, 2003); Section 10, Atomic, Molecula and Optical Physics; Ionizatoin Potentials of Atoms and Atomic Ions

[7] M. Agostini, S. Appel, G. Bellini et al. (Borexino Collaboration), Phys. Rev. D 92, 031101 (2015); arXiv:1506.04610 [hep-ex]

[8] A. Gando, Y. Gando, H. Hanakago et al. (KamLAND Collaboration), Phys. Rev. D 88, 033001 (2013); arXiv:1303.4667v2

[9] J. H. Davies and D. R. Davies, Solid Earth 1, 5 (2010)

[10] J. Hansen, M. Sato, P. Kharecha and K. von Schuckmann, Atmos. Chem. Phys. 11, 13421 (2011)

[11] Langseth, M. G., S. P. Clark Jr., J. L. Chute, S. J. Keihm and A. E. Wechsler, The Moon 4, 390 (1972)

[12] V. D. Krotikov and V. S. Troitsky, Soviet J. Phys. Uspekhi 6, 841 (1964)

[13] Paige, D. A., et al., Space Sci. Rev. 150, 125 (2010)

[14] Watson, K., J. Geophys. Rev. 72, 331 (1967)

[15] V. V. Sinev, L. B. Bezrukov et al., Phys. Particles and Nuclei 46, 186 (2015)

[16] V. V. Sinev, L. B. Bezrukov, E. A. Litvinovich, I. M. Machulin, M. D. Skorokhvatov, S. V. Sukhotin, Phys. Part. Nucl. 46, 186 (2015) 